\title{
Communication
}

\section{High-Sensitive TRBC1-Based Flow Cytometric Assessment of T-Cell Clonality in T $\alpha \beta$-Large Granular Lymphocytic Leukemia}

\author{
Noemí Muñoz-García ${ }^{1,2}$, F. Javier Morán-Plata ${ }^{1,2}$, Neus Villamor ${ }^{2,3}$, , Margarida Lima ${ }^{4,5, \dagger}$, Susana Barrena ${ }^{1,2}$, \\ Sheila Mateos ${ }^{1,2}$, Carolina Caldas ${ }^{1,2}$, Jacques J. M. van Dongen ${ }^{1,6}\left(\mathbb{D}\right.$, Alberto Orfao ${ }^{1,2, \pm(1)}$ \\ and Julia Almeida ${ }^{1,2, *, \mp(1)}$
}

check for updates

Citation: Muñoz-García, N.; Morán-Plata, F.J.; Villamor, N.; Lima, M.; Barrena, S.; Mateos, S.; Caldas, C.; van Dongen, J.J.M.; Orfao, A.; Almeida, J. High-Sensitive TRBC1-Based Flow Cytometric Assessment of T-Cell Clonality in T $\alpha \beta$-Large Granular Lymphocytic Leukemia. Cancers 2022, 14, 408. https://doi.org/10.3390/ cancers14020408

Academic Editor: Alberto Maria Martelli

Received: 16 December 2021 Accepted: 10 January 2022 Published: 14 January 2022 Publisher's Note: MDPI stays neutral with regard to jurisdictional claims in published maps and institutional affiliations.

Copyright: (C) 2022 by the authors. Licensee MDPI, Basel, Switzerland. This article is an open access article distributed under the terms and conditions of the Creative Commons Attribution (CC BY) license (https:// creativecommons.org/licenses/by/ $4.0 /)$.
1 Translational and Clinical Research Program, Centro de Investigación del Cáncer and IBMCC (CSIC - University of Salamanca), Cytometry Service, NUCLEUS, Department of Medicine, University of Salamanca (USAL) and Institute of Biomedical Research of Salamanca (IBSAL), 37007 Salamanca, Spain; noemimg@usal.es (N.M.-G.); fjmoranp@usal.es (F.J.M.-P.); subadelfa@usal.es (S.B.); sheilamateos@usal.es (S.M.); carolina.caldas@usal.es (C.C.); J.J.M.van_Dongen@lumc.nl (J.J.M.v.D.); orfao@usal.es (A.O.)

2 Biomedical Research Networking Centre Consortium of Oncology (CIBERONC), Instituto de Salud Carlos III, 28029 Madrid, Spain; villamor@clinic.cat

3 Department of Pathology, Hematopathology Unit, Hospital Clínic, IDIBAPS, 08036 Barcelona, Spain

4 Department of Hematology, Laboratory of Cytometry, Hospital de Santo António, Centro Hospitalar do Porto, 4099-001 Porto, Portugal

5 Unit for Multidisciplinary Research in Biomedicine (UMIB), Abel Salazar Institute of Biomedical Sciences (ICBAS), University of Porto, 4050-313 Porto, Portugal

6 Department of Immunology, Leiden University Medical Center (LUMC), 2333 Leiden, The Netherlands

* Correspondence: jalmeida@usal.es; Tel.: +34-923-29-48-11 (ext. 5816)

† Sadly, M. Lima passed away on 1 November 2021. As she had significantly contributed to the study, the authors decided to submit the paper with her name as a co-author.

$\ddagger \quad$ These authors contributed equally to this work.

Simple Summary: TRBC1 expression analysis by flow cytometry (FCM) has been recently proved to be a useful, simple and fast approach to assessing T $\alpha \beta$-cell clonality. The aim of this study was to validate the utility of this assay specifically for the diagnosis of T-cell clonality of T-large granular lymphocytic leukemias (T-LGLL), as more mature polyclonal $\mathrm{T} \alpha \beta$ large granular lymphocytes $\left(\mathrm{T} \alpha \beta\right.$-LGL) show broader TRBC $1^{+} / \mathrm{TRBC}^{-}$ratios vs. total T $\alpha \beta$ cells. Our results showed that a TRBC1-FCM assay is also a fast and easy method for detecting T-cell clonality in T-LGLL based on altered (increased or decreased) percentages of TRBC1 ${ }^{+} \mathrm{T} \alpha \beta$ cells of LGL expansions (i.e., with lymphocytosis) suspected of T-LGLL, whereas in the absence of lymphocytosis (or in T $\alpha \beta C D 4-L G L L)$, the detection of increased absolute cell-counts of more precisely defined subpopulations of T-LGL expressing individual TCRV $\beta$ families is required.

Abstract: Flow cytometric (FCM) analysis of the constant region 1 of the T-cell receptor $\beta$ chain (TRBC1) expression for assessing $T \alpha \beta$-cell clonality has been recently validated. However, its utility for the diagnosis of clonality of T-large granular lymphocytic leukemia (T-LGLL) needs to be confirmed, since more mature T $\alpha \beta$ cells (i.e., T-LGL normal-counterpart) show broader TRBC1 ${ }^{+} / \mathrm{TRBC}^{-}$ ratios vs. total $\mathrm{T} \alpha \beta$ cells. We compared the distribution and absolute counts of $\mathrm{TRBC} 1^{+}$and $\mathrm{TRBC} 1^{-}$ $\mathrm{T} \alpha \beta$-LGL in blood containing polyclonal $(n=25)$ vs. clonal $(n=29)$ LGL. Overall, polyclonal TRBC1 ${ }^{+}$ or TRBC $1^{-}$T $\alpha \beta$-LGL ranged between 0.36 and 571 cells $/ \mu \mathrm{L}\left(3.2-91 \% \mathrm{TRBC}^{+}\right.$cells), whereas the clonal LGL cases showed between 51 and 11,678 cells $/ \mu \mathrm{L}\left(<0.9 \%\right.$ or $>96 \% \mathrm{TRBC}^{+}$cells $)$. Among the distinct TCRV $\beta$ families, the CD28- effector-memory and terminal-effector polyclonal T $\alpha \beta$ cells ranged between 0 and $25 \mathrm{TRBC}^{+}$or TRBC $1^{-}$cells $/ \mu \mathrm{L}$ and between 0 and $100 \% \mathrm{TRBC} 1^{+}$cells, while clonal LGL ranged between 32 and $5515 \mathrm{TRBC}^{+}$or $\mathrm{TRBC} 1^{-}$cells $/ \mu \mathrm{L}$, representing $<1.6 \%$ or $>98 \%$ $\mathrm{TRBC}^{+}$cells. Our data support the utility of the TRBC1-FCM assay for detecting T-cell clonality in expansions of T $\alpha \beta$-LGL suspected of T-LGLL based on altered percentages of TRBC $1^{+} \mathrm{T} \alpha \beta$ cells. However, in the absence of lymphocytosis or in the case of T $\alpha \beta C D 4$-LGL expansion, the detection of increased absolute cell counts by the TRBC1-FCM assay for more accurately defined subpopulations 
of T $\alpha \beta$-LGL-expressing individual TCRV $\beta$ families, allows the detection of T-cell clonality, even in the absence of phenotypic aberrations.

Keywords: TRBC1; JOVI-1; TCRV $\beta$ repertoire; large granular lymphocytic leukemia; LGLL; large granular lymphocytes; LGL; T $\alpha \beta$-cell maturation stages; T $\alpha \beta$ effector cells; flow cytometry T-cell clonality assessment

\section{Introduction}

T-cell large granular lymphocytic leukemia (T-LGLL) is a rare chronic lymphoproliferative disorder (CLPD) characterized by the clonal expansion of mature cytotoxic $\mathrm{T}$ cell-i.e., large granular T lymphocytes (T-LGL) - in blood and potentially also in other tissues [1-3]. A threshold of $2 \times 10^{9} \mathrm{~T}-\mathrm{LGL} / \mathrm{L}$ of blood was initially mandatory for the diagnosis of T-LGLL, but lower LGL counts of $\geq 0.5-2 \times 10^{9}$ cells/L are currently also recognized as a diagnostic criterion for T-LGLL when associated with typical clinical manifestations of the disease (i.e., cytopenias or autoimmune disorders) [4-7]. Altogether, this indicates that no single T-LGL count cut-off allows for clear-cut discrimination between neoplastic and reactive expansions of T-LGL. In turn, T-LGLL cells frequently display morphological and phenotypical features that largely overlap with their normal counterpart of (activated) terminal effector (TE), and to a lesser extent, also CD28 ${ }^{-}$effector memory (EM) T cells [5,8]. Further, more genetic surrogate markers of a clonal disorder, such as STAT3 and STAT5B mutations, have been reported in only a fraction of between $21 \%$ and $73 \%$ of all T-LGLL patients [8-10]. Considering all the above, the demonstration of the clonal nature of the expanded T-LGL in the diagnostic work-up of T-LGLL still remains a challenge, particularly in cases presenting without lymphocytosis $[8,11]$.

The evaluation of the pattern of expression of the constant region 1 of the T-cell receptor $\beta$ chain (TRBC1) by flow cytometry (FCM) has been recently demonstrated to be a fast, simple, specific and accurate method for assessment of the clonal nature of $T \alpha \beta$ cells in patients suspected of having T-CLPD [12-15]. Thus, the monoclonal expansions of $\mathrm{T} \alpha \beta$ cells typically show a monotypic/unimodal TRBC1 expression profile (either TRBC1 ${ }^{+}$ or TRBC $1^{-}$), whereas normal/reactive T $\alpha \beta$ cells consist of a (bimodal) admixture of both $\mathrm{TRBC}^{+}$and TRBC1 $1^{-}$cells $[12,13,16]$, where TRBC $1^{+}$cells represent nearly $40 \% \pm 6.7 \%$ (mean $\pm 1 \mathrm{SD}$ ) of all T $\alpha \beta$ cells [13]. However, recent data also indicate that the percentage of TRBC1 $1^{+} \mathrm{T} \alpha \beta$ cells may vary substantially among the different maturation-associated compartments of $\mathrm{T}$ cells. Thus, more mature populations of EM (particularly within the $\mathrm{CD} 28^{-}$subset) early-effector (EE) and TE-T cells display significantly broader ranges of $\mathrm{TRBC}^{+}$and $\mathrm{TRBC}^{-}$cells, compared to both total $\mathrm{T} \alpha \beta$ cells and their naïve and central/transitional memory compartments [13]. Of note, clonal cells from T-LGLL patients, as well as most small T-LGL clones detected in otherwise healthy donors (HDc) [14,15], frequently display phenotypic profiles that match those of EM/TE cells [5,8]. Despite this, the recent evidence showing that broader percentages of TRBC1 $1^{+}$and TRBC1 $1^{-}$cells exist among more mature polyclonal T $\alpha \beta$-LGL cells has not been taken into account in applying the TRBC1-FCM approach for the identification of clonal LGL cells. Here, we validated the utility of the TRBC1-FCM assay for the assessment of T $\alpha \beta$-cell clonality in T-LGLL via direct comparisons with the relative distribution and absolute counts of $\mathrm{TRBC} 1^{+}$and TRBC1 ${ }^{-}$T $\alpha \beta$ cells among normal T $\alpha \beta$-LGL.

\section{Materials and Methods}

\subsection{Patients, Controls and Samples}

A total of 54 EDTA-anticoagulated whole peripheral blood (PB) samples (from an identical number of subjects) were studied. Forty-two of them corresponded to samples included in a previously reported study [13], while the remaining 12 samples were prospectively collected between July and September 2021. From all PB samples, 17 corresponded 
to adult HD, 5 to HDc (identified for the first time in this study by the TRBC1-based FCM assay), 8 were collected from subjects with reactive T $\alpha \beta$ lymphocytosis and 24 corresponded to patients with T $\alpha \beta$-LGLL (18 T $\alpha \beta C D 8-L G L L, 5$ T $\alpha \beta C D 4-L G L L$ and 1 T $\alpha \beta$ double-negative LGLL). Additionally, residual polyclonal T $\alpha \beta$ cells from HDc and LGLL cases in which these could be clearly distinguished from the clonal T $\alpha \beta$ cells were also analyzed as reactive T $\alpha \beta$ cells. The mean age $( \pm 1 S D)$ of subjects at sample collection was of $38 \pm 8$ years $(y), 72 \pm 14 \mathrm{y}, 55 \pm 19 \mathrm{y}$ and $66 \pm 17 \mathrm{y}$ for $\mathrm{HD}$, individuals with reactive T $\alpha \beta$-LGL, HDc and patients with T $\alpha \beta$-LGLL, respectively.

Prior to the study, all patients and controls gave their written informed consent to participate, and the study was conducted according to the guidelines of the Declaration of Helsinki after it had been approved by the local institutional Ethics Committee (University Hospital of Salamanca/IBSAL, CEIm reference number PI 202012 643).

\subsection{Immunophenotypic Stainings}

All samples were immunophenotyped using a direct immunofluorescence stain-andthen-lyse technique based on the EuroFlow standard operating procedures (SOP) [17-19]. The samples included in the previous publication [13] were stained with the anti-TRBC1 antibody (clone JOVI-1) in combination with monoclonal antibodies (Mab) recognizing maturation markers (e.g., CD27, CD28, CD45RA and CD62L; Table S1 Panel I), strictly following the EuroFlow SOP. The other samples were stained with a total of 40 different Mab reagents, including: (i) a common backbone of 12 antibodies against TRBC1, maturationassociated molecules (CD27, CD28, CD45RA and CD62L), markers which are frequently aberrantly expressed by clonal LGL (i.e., CD2, CD5 and CD7), and a set of four markers for identification of T cells and their major subsets (CD3, CD4, CD8 and TCR $\gamma \delta$ ); (ii) $24 \mathrm{TCRV} \beta$ Mab reagents (IOTest ${ }^{\circledR}$ Beta Mark TCRV $\beta$ Repertoire Kit-Beckman-Coulter, Brea, CA, USA); and (iii) CD45 conjugated with four different fluorochromes (Table S1 Panel II). A detailed description of the staining protocol (adapted from the EuroFlow reference) [17-19] aimed at combining all these reagents into only two antibody combinations, ready to be measured in the flow cytometer, is provided in the Supplementary Materials, together with the precise composition of the antibody reagent panel (Protocol S1 and Table S1 Panel II). The sources and specificities of all Mab reagents used for the immunophenotypic assays described above are detailed in Table S2.

Immediately after the completion of sample preparation, stained cells were measured in a 5-laser Cytek Aurora flow cytometer (Cytek Biosciences, Fremont, CA, USA) using SpectroFlo software (version 2.1.0, Cytek Biosciences, Fremont, CA, USA). Instrument setup, calibration and daily quality control, as well as monitoring, were performed according to well-established EuroFlow protocols [18,19]. For data analysis, Infinicyt ${ }^{\mathrm{TM}}$ software (version 2.0, Cytognos, Salamanca, Spain) was used. A minimum of 500,000 target cells was acquired per sample, and $\geq 10$ clustered cellular events were required for the definition of a cell population.

\subsection{Calculation of the Number of TRBC1 ${ }^{+}$and $\mathrm{TRBC1}^{-}$Cells within Different}

Maturation-Associated Compartments of T $\alpha \beta$ Cells and Their Major TCD8 and TCD4 Subsets

The percentage and absolute number of $\mathrm{TRBC}^{+}$and $\mathrm{TRBC}^{-}{ }^{-}$cells within each of the different maturation-associated (i.e., naïve, central memory, transitional memory, CD28 ${ }^{+}$ and CD28- EM, EE and TE) populations of T $\alpha \beta$ cells and their major TCD8 and TCD4 subsets was investigated as previously described [13].

In addition, the percentage and absolute count of $\mathrm{TRBC}^{+}$and $\mathrm{TRBC} 1^{-} \mathrm{T} \alpha \beta$ cells within each maturation stage was also calculated for $\mathrm{T} \alpha \beta$ cells expressing each individual TCRV $\beta$ family.

\subsection{Assessment of T-Cell Clonality on FACS-Sorted Cell Populations}

The clonal nature of T-cell populations was confirmed in FACS-sorted cells highly purified ( $\geq 95 \%$ purity) by $3-5 \mathrm{~mL}$ of whole blood using a BD FACSAria-III flow cytometer 
(Becton Dickinson Biosciences, San Jose, CA, USA) [20-22] based on the presence of a single or a few dominant TRB and/or TRG gene rearrangements for the clonal cell subsets. In 2 T-LGLL patients, the T-cell clonality was further established on the purified cells through the confirmation of the presence of a STAT3 mutation [2,9].

\section{Results and Discussion}

In the first step, we investigated the TRBC1 expression profile of the aberrant/ pathological cells from T-LGLL $(n=20)$ and HDc $(n=3)$ with that of the polyclonal EM-EE-TE T cells from HD $(n=11)$ and patients with reactive conditions $(n=8)$ in a total of 42 blood samples. Overall, the absolute count of the polyclonal TRBC1 ${ }^{+}$and $\mathrm{TRBC} 1^{-}$ $\mathrm{EM}+\mathrm{EE}+\mathrm{TE} \mathrm{T} \alpha \beta$ cells ranged between 0.36 and 225 cells $/ \mu \mathrm{L}$ in $\mathrm{HD}$ and between 0.71 and 571 cells $/ \mu \mathrm{L}$ in reactive cases. In turn, clonal T $\alpha \beta-\mathrm{LGL}$, as identified by the TRBC1-FCM assay, ranged between 51 and 610 cells $/ \mu \mathrm{L}$ in HDc and between 137 and 11,678 cells $/ \mu \mathrm{L}$ in T-LGLL (Figure 1A). As expected, the absolute number of T $\alpha \beta$-LGL from most T-LGLL $(11 / 17,65 \%)$ were significantly $(p<0.0001)$ higher than those of EM + EE + TE cells from $\mathrm{HD}$ and reactive cases, allowing for a clear-cut diagnosis of T-LGLL. However, while this was true for $10 / 11$ (91\%) T $\alpha \beta C D 8-L G L L$ patients, in only $1 / 3(33 \%)$ of T $\alpha \beta C D 8-H D c$ cases and $1 / 5(20 \%)$ of T $\alpha \beta C D 4-L G L L$ patients, the absolute blood count of either TRBC $1^{+}$or TRBC1 ${ }^{-}$clonal T-LGL outnumbered the counts observed among their normal/reactive (mature EM + EE + TE) cell counterpart in normal/reactive blood (Figure 1A,C,E). Based on these findings, it may be concluded that the absolute count of pathological T-LGL cells identified by the TRBC1 expression profile within the whole population of T $\alpha \beta$ cells cannot be used as a universal (single) criterion for clear-cut detection of clonal T $\alpha \beta$-LGL, particularly in T-LGLL cases that present without lymphocytosis and/or that carry relatively small $(<2000$ cells $/ \mu L)$ T $\alpha \beta C D 4-L G L$ or T $\alpha \beta C D 8-L G L$ clones in the blood $[4,23]$. In contrast, once the percentage of $\mathrm{TRBC}^{+}$cells among all aberrant/pathological vs. normal/reactive EM and TE T $\alpha \beta$ cells was considered, cases carrying clonal cells could be unequivocally identified and distinguished from those that only had normal polyclonal cells for all T-LGLL and HDc subjects analyzed (Figure 1B). Thus, the percentage of $\mathrm{TRBC}^{+}$cells among the more mature compartments of EM and TE T $\alpha \beta$ cells ranged between $7.2 \%$ and $91 \%$ in HD and between $3.2 \%$ and $89 \%$ in reactive cases, while in all HDc and T-LGLL cases, clonal cells accounted for a more extreme percentage of $\mathrm{TRBC}^{+}$cells (either $<0.9 \%$ or $>96 \%$ ) within the corresponding maturation-associated compartment of $\mathrm{T}$ cells containing the expanded/suspicious (pathological) cell fraction (Figure 1B). Further subclassification of $\mathrm{T}$ cells into their major T $\alpha \beta C D 8$ and $\mathrm{T} \alpha \beta \mathrm{CD} 4$ subsets confirmed the presence of clonal cells in all T $\alpha \beta C D 8$ LGLL and HDc cases based on the percentage of TRBC $1^{+}$cells within their corresponding maturation-associated T-cell subset, once compared with their distribution in normal/reactive blood: all T $\alpha \beta C D 8-L G L L$ showed either lower $(<1.3 \%)$ or higher $(>97.2 \%)$ percentages of TRBC $1^{+}$TE cells than normal HD (7.4-96\%) and reactive (4.7-89\%) EM + EE + TE cells (Figure 1D). In contrast, the fraction of $\mathrm{TRBC}^{+}$cells from all T $\alpha \beta C D 4-L G L L$ (either $<0.47 \%$ or $>92 \%$ of suspicious cells) still overlapped with that of polyclonal T $\alpha \beta C D 4$ CD28 $8^{-}$EM or TE T cells in $4 / 5(80 \%)$ reactive cases investigated (Figure 1F). 


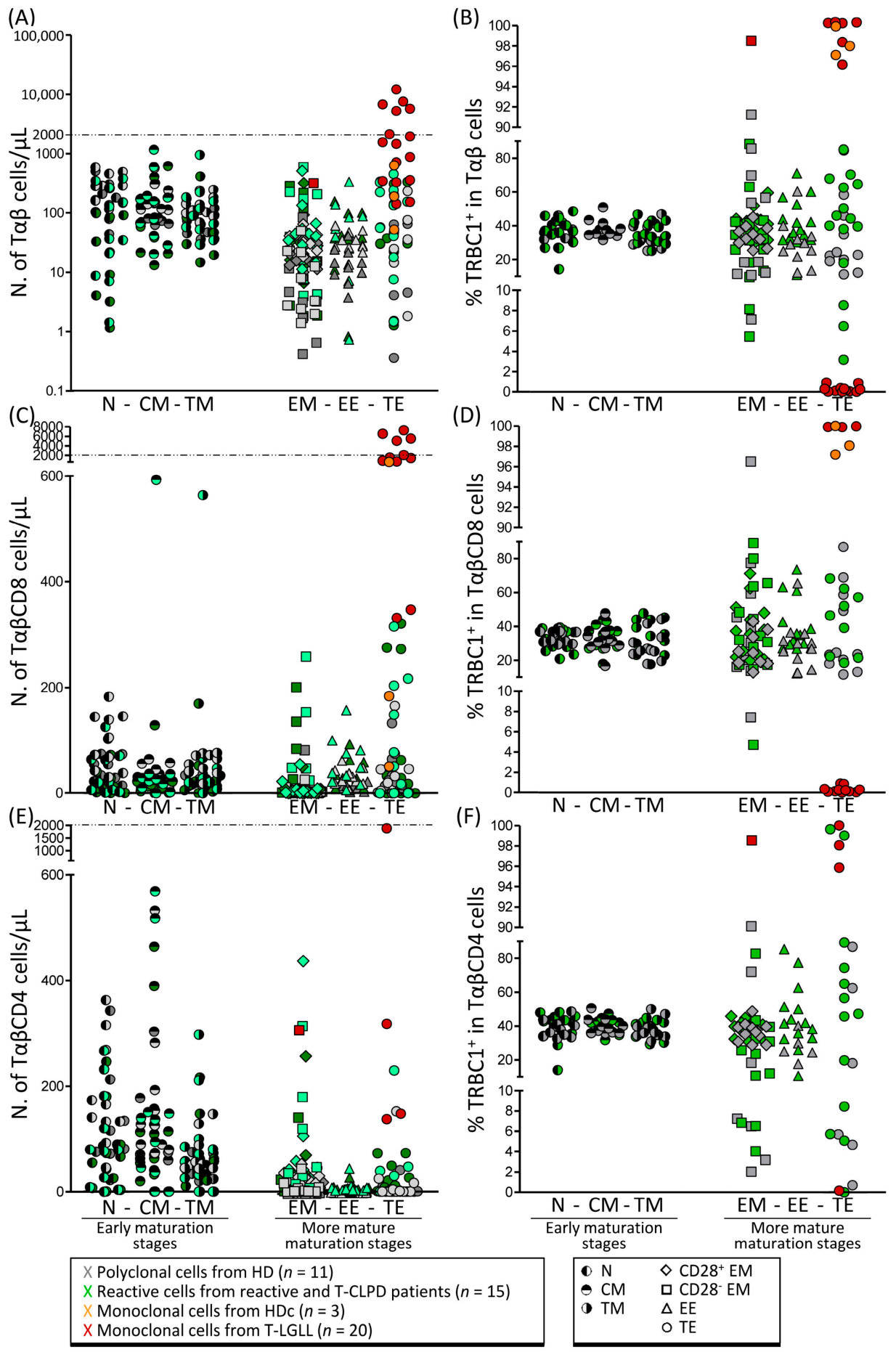

Figure 1. Absolute counts and relative distribution of $\mathrm{TRBC}^{+}$and $\mathrm{TRBC}^{-}$cells among nor$\mathrm{mal} /$ reactive (polyclonal) $\mathrm{T} \alpha \beta$-cell populations from normal/reactive blood classified according to their maturation stage vs. clonal T $\alpha \beta$-LGL. Absolute $(\mathbf{A}, \mathbf{C}, \mathbf{E})$ and relative $(\mathbf{B}, \mathbf{D}, \mathbf{F})$ number of TRBC1 ${ }^{+}$ (dark color) and $\mathrm{TRBC}^{-}$(light color) cells identified among normal (gray dots) and reactive (green dots) populations of (polyclonal) total T $\alpha \beta$ cells (A,B), T $\alpha \beta C D 8$ cells (C,D) and T $\alpha \beta C D 4$ cells (E,F), classified according to their maturation stage, compared to clonal T $\alpha \beta$-LGL from HDc (orange dots) and T-LGLL (brown dots). Abbreviations (alphabetical order): CM, central memory; EE, early effector; EM, effector memory; HD, healthy donor; HDc, healthy donor with a T $\alpha \beta$ clone; LGL, large granular lymphocyte; N, naïve; N., number; PB, peripheral blood; T-CLPD, chronic lymphoproliferative disorder of T cells; T-LGLL, T-cell large granular lymphocytic leukemia; TE, terminal effector; TM, transitional memory. 
In order to better define the utility of the TRBC1-FCM approach for high-sensitive assessment of T-cell clonality in T-LGLL in the clinical settings and improve its specificity for detection of (small) LGL clones, we subsequently combined the TRBC1 assay with the analysis of the TCRV $\beta$ repertoire and/or the presence of aberrant phenotypes by FCM. For this purpose, a second series of PB samples from 12 individuals (six HD, two T $\alpha \beta C D 8$ HDc and four T $\alpha \beta C D 8$-LGLL) was studied. Importantly, the simultaneous staining for T-cell maturation-associated markers and the TCRV $\beta$ repertoire allowed for the first time the detailed analysis of the TRBC1 expression profile together with the distribution of the different TCRV $\beta$ families, among normal/reactive CD28 ${ }^{-} \mathrm{EM}$ and TE T $\alpha \beta$ cells. It should be noted that normal T $\alpha \beta$ cells and their major subpopulations of $\mathrm{T} \alpha \beta \mathrm{CD} 4$ and T $\alpha \beta C D 8$ cells are known to display a variable distribution for the different TCRV $\beta$ families by FCM, and the TCRV $\beta$ antibodies included in the kit used here cover approximately $70-75 \%$ of the whole TCRV $\beta$ repertoire [24]. As expected, the more mature CD28- EM and TE compartments of blood T cells showed a more restricted TCRV $\beta$ repertoire than total $\mathrm{T} \alpha \beta$ and their major $\mathrm{T} \alpha \beta \mathrm{CD} 8$ and $\mathrm{T} \alpha \beta \mathrm{CD} 4$ cell subsets (Figure S1). The narrower TCRV $\beta$ repertoire observed for the more mature compartments of blood T $\alpha \beta$ cells is most likely due to the accumulation of effector T cells with a more restricted TCR repertoire, which is specific to a relatively limited number of antigens that these cells have recently encountered at these stages of maturation, in line with previous observations reported on activated T cells [25]. Further subsetting of mature CD28- EM and TE T $\alpha \beta$ cells expressing individual TCRV $\beta$ families into TRBC1 $1^{+}$and TRBC1 ${ }^{-}$cells showed significantly higher absolute counts of clonal TRBC1 $1^{+}$or TRBC1 ${ }^{-}$T $\alpha \beta$ and T $\alpha \beta C D 8$ cells in T-LGLL and HDc (32-5515 cells $/ \mu \mathrm{L}$ ) compared to those identified among polyclonal TRBC $1^{+}$and TRBC1 ${ }^{-}$total T $\alpha \beta(0-25$ cells $/ \mu \mathrm{L})$ and T $\alpha \beta C D 8(0-21$ cells $/ \mu \mathrm{L})$ cells from normal/reactive blood (Figure $2 \mathrm{~A}, \mathrm{C}$ ). Therefore, the absolute number of cells identified by a combination of markers that more precisely defines the TRBC1 expression profile within T $\alpha \beta$ cells expressing a given TCRV $\beta$ family (within a specific maturation stage) provides higher sensitivity and specificity for the detection of clonal $\mathrm{T}$ cells and improves the accuracy of the classical TRBC1-FCM approach for the detection of clonal T $\alpha \beta$-LGL.

Subsequent analysis of the percentage of TRBC1 $1^{+}$cells per TCRV $\beta$ family within polyclonal LGL (Figure 2B,D) revealed that a polytypic/bimodal TRBC1 expression pattern was preserved for each TCRV $\beta$ family among the total T $\alpha \beta$ cells (range: $38-55 \%$ ) $[13,14]$. In contrast, highly variable and more extreme $\mathrm{TRBC}^{+}$percentages were observed when the analysis was restricted to the more mature $\left(\mathrm{CD} 28^{-} \mathrm{EM}\right.$ and TE) compartments of total $\mathrm{T} \alpha \beta$ and T $\alpha \beta C D 8$ normal/reactive cells expressing individual TCRV $\beta$ families (range: $0 \%$ to $100 \%$ for all TCRV $\beta$ families, except for TCRV $\beta 5.3^{+}$cells within the total T $\alpha \beta$ cells and the TCRV $\beta 5.3^{+}, \mathrm{TCRV} \beta 7.2^{+}, \mathrm{TCRV} \beta 13.2^{+}$and TCRV $\beta 14^{+}$cells among T $\alpha \beta C D 8$ cells). This translated into a substantial overlap, with the percentage of TRBC $1^{+}$cells observed for clonal T $\alpha \beta$-LGL (either $<1.6 \%$ or $>97.7 \%$ of TRBC $1^{+}$cells; Figure $2 \mathrm{~B}, \mathrm{D}$ ). However, it should be noted that clonal T $\alpha \beta$-LGL from half (3/6) of the T-LGLL/HDc investigated showed an aberrant phenotype that would allow their unequivocal identification and discrimination from residual polyclonal $\mathrm{T}$ cells (Table S3). These results indicate that the identification of clonality among T $\alpha \beta$-LGL based on the pattern of expression of TRBC1 among cells expressing a single TCRV $\beta$ family should be based on the combined assessment of the absolute cell counts and/or the expression of aberrant phenotypes. 

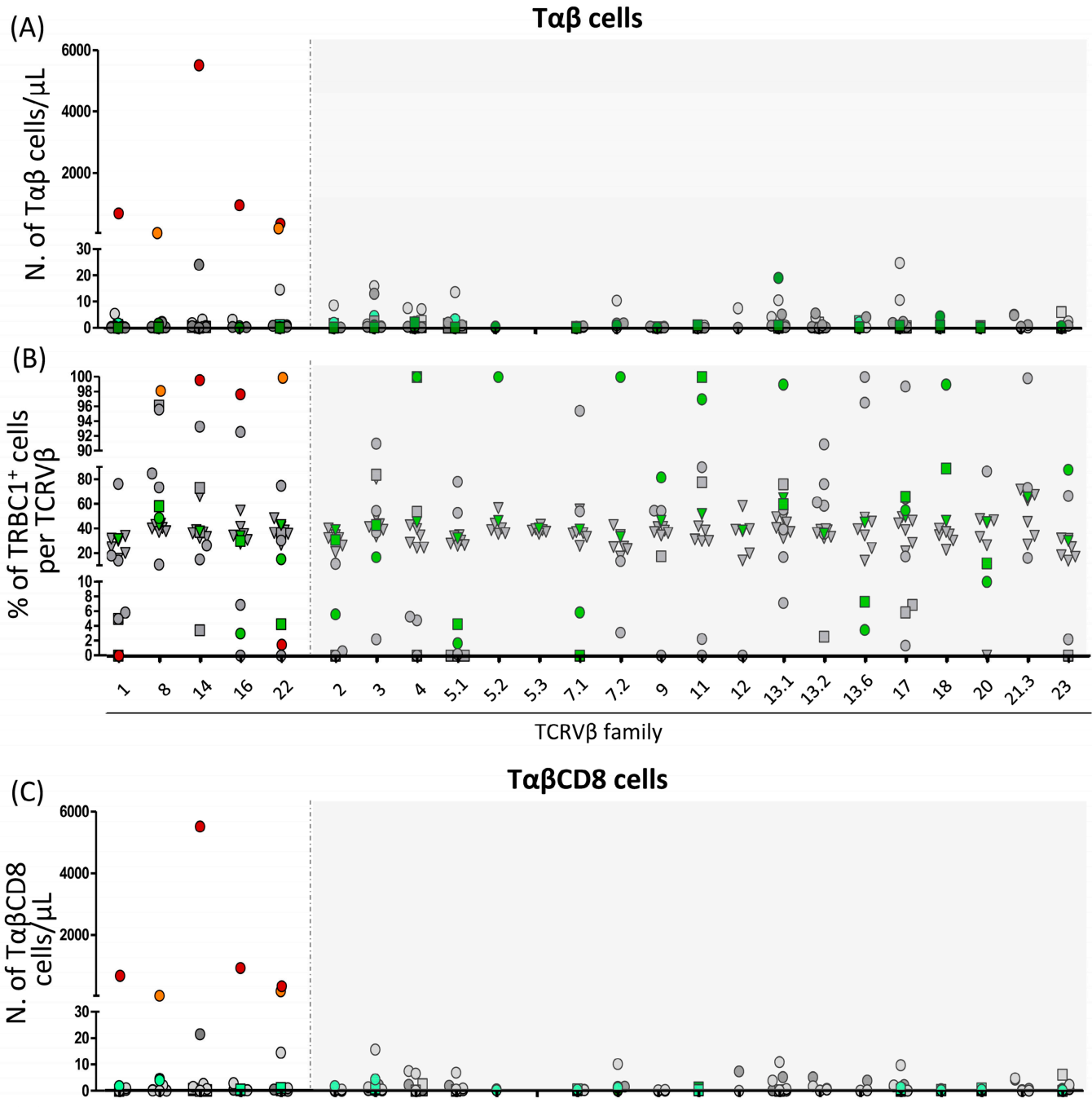

(D)

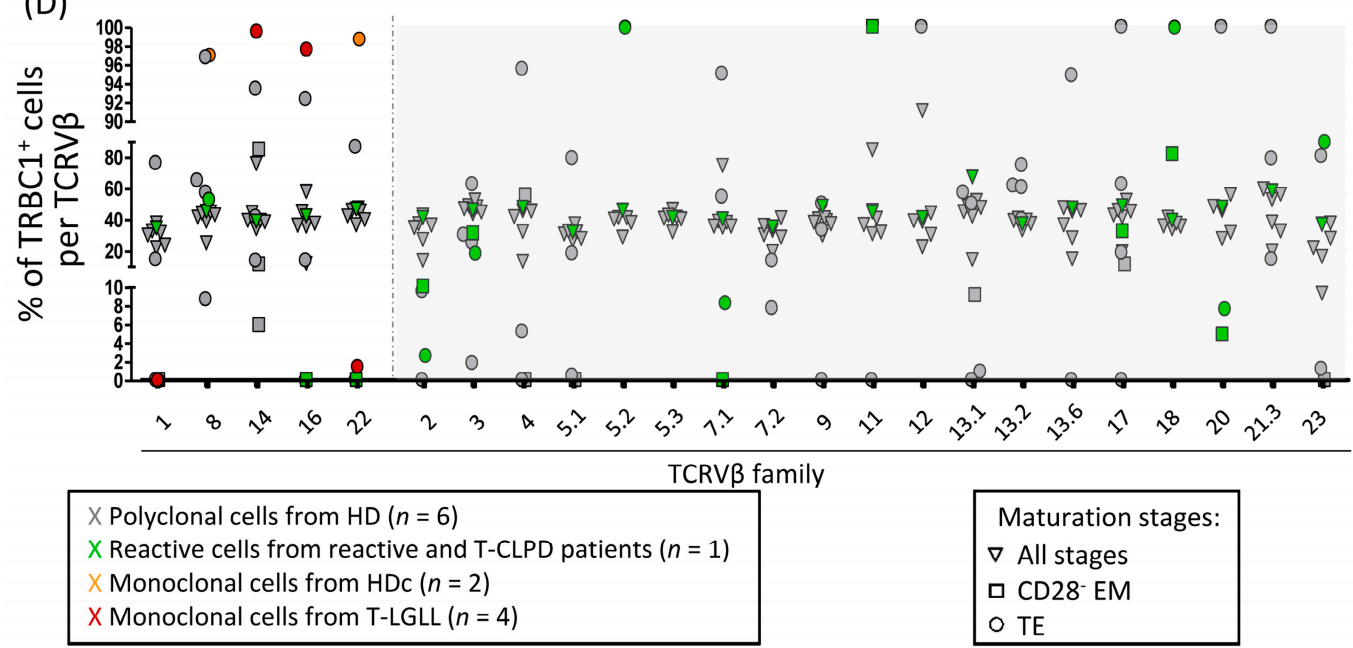

Figure 2. Absolute counts and relative distribution of TRBC $1^{+}$cells per TCRV $\beta$ family, among the more mature subsets (CD28- EM and TE) of normal $(n=6)$ and reactive $(n=1)$ polyclonal T $\alpha \beta$-cells vs. clonal T $\alpha \beta$-LGL ( $n=2$ HDc and $n=4$ LGLL). Absolute number $(\mathbf{A}, \mathbf{C})$ and percentage $(\mathbf{B}, \mathbf{D})$ of $\mathrm{TRBC}^{+}$cells within each TCRV $\beta$ family among total $\mathrm{T} \alpha \beta$ cells $(\mathbf{A}, \mathbf{B})$ and their major T $\alpha \beta C D 8$ subset (C,D). Abbreviations (alphabetical order): EM, effector memory; HD, healthy donor; HDc, healthy donor with a T-LGL clone; N., number; T-CLPD, chronic lymphoproliferative disorder of T cells; T-LGLL, T-cell large granular lymphocytic leukemia; TE, terminal effector. 


\section{Conclusions}

Here, we confirm and expand on previous observations about the clinical utility of the TRBC1-FCM assay for the rapid and easy assessment of T-cell clonality in the blood of individuals presenting with LGL lymphocytosis who are suspicious of T-LGLL based on the demonstration of altered (increased or decreased) percentages of $\mathrm{TRBC} 1^{+} \mathrm{T} \alpha \beta$ cells (around either 0 or $100 \%$ ). In contrast, our data indicate that, in the absence of lymphocytosis (or if T $\alpha \beta C D 4-L G L L$ is suspected), the detection of increased absolute cell counts of subpopulations of T-LGL expressing individual TCRV $\beta$ families is required for the detection of T-cell clonality by the TRBC1-FCM assay; therefore, a more precise definition of T-LGL populations by TCRV $\beta$ provides the bases for the highly sensitive detection of T-cell clonality by FCM, even in the absence of aberrant phenotypes. Further comparisons of the TRBC1 assay here proposed against conventional T-cell clonality molecular approaches in a larger series of T-LGLL/HDc vs. normal/reactive PB samples covering the whole TCRV $\beta$ repertoire are required to confirm our preliminary findings.

Supplementary Materials: The following are available online at https:/ /www.mdpi.com/article/10 $.3390 /$ cancers14020408/s1: Protocol S1: Combining sample aliquots stained with a CD45 antibody conjugated to 8 different fluorochromes into only two antibody combinations ready to be measured in the flow cytometer, Table S1: Panels of fluorochrome-conjugated antibody reagents used in this study, Table S2: Sources and specificities of the monoclonal antibody reagents used in this study, Table S3: Detailed immunophenotypic features of T-cell subsets showing extreme TRBC1 ${ }^{+}$percentages within the more mature polyclonal and monoclonal T $\alpha \beta$-cell populations expressing a specific TCRV $\beta$ family, Figure S1: Distribution of T cells expressing different TCRV $\beta$ families among total T $\alpha \beta$ cells and their $\mathrm{T} \alpha \beta \mathrm{CD} 8^{+}$and $\mathrm{T} \alpha \beta \mathrm{CD} 4^{+}$cell subsets and their maturation-associated stages of $\mathrm{CD} 28^{-}$ effector memory and terminal effector cells as identified in the blood of healthy donors $(n=6)$.

Author Contributions: J.A., A.O. and J.J.M.v.D. contributed to the conceptualization, design and supervision of the study, as well as to the recruitment of funding. N.M.-G., F.J.M.-P., N.V., M.L., S.M. and C.C. performed the experiments and validation studies, as well as the data analysis and interpretation. N.M.-G. coordinated the planning and execution of the experiments. N.M.-G., N.V., M.L. and S.B. selected cases for the study and collected their relevant clinical information; N.M.-G., A.O. and J.A. wrote the manuscript. All authors have read and agreed to the published version of the manuscript.

Funding: This work was supported by the CB16/12/00400 (CIBERONC) and PI20-01346 grants from the Instituto de Salud Carlos III, Ministerio de Ciencia e Innovación (Madrid, Spain) and FONDOS FEDER; the 0639-IDIAL-NET-3-3 grant (INTERREG POCTEP Spain-Portugal) from Fondo Europeo de Desarrollo Regional, and by the EuroFlow Foundation (Leiden, The Netherlands). N.M.-G. was supported by a pre-doctoral grant (Ref. IBPredoc17/00012) from IBSAL (Salamanca, Spain). M.L., N.V., J.J.M.v.D., A.O. and J.A. are members of the EuroFlow Consortium, www.euroflow.org (accessed on 16 December 2021).

Institutional Review Board Statement: All the patients and controls gave their written informed consent to participate in the study, and the project was conducted according to the guidelines of the Declaration of Helsinki and approved by the local institutional Ethics Committees (University Hospital of Salamanca/IBSAL, CEIm reference number PI 202012 643).

Informed Consent Statement: Informed consent was obtained from all subjects involved in the study.

Data Availability Statement: The data presented in this study are available in this article (and Supplementary Materials).

Conflicts of Interest: The authors declare no competing financial interest or any other conflict of interest. 


\section{References}

1. Loughran, T.P. Clonal diseases of large granular lymphocytes. Blood 1993, 82, 1-14. [CrossRef]

2. Swerdlow, S.H.; Campo, E.; Harris, N.L.; Jaffe, E.S.; Pileri, S.A.; Stein, H.; Thiele, J.; Vardiman, J.M. WHO Classification of Tumours of Haematopoietic and Lymphoid Tissues, 4th ed.; WHO Press: Lyon, France, 2017.

3. Semenzato, G.; Zambello, R.; Starkebaum, G.; Oshimi, K.; Loughran, T.P. The lymphoproliferative disease of granular lymphocytes: Updated criteria for diagnosis. Blood 1997, 89, 256-260. [CrossRef] [PubMed]

4. Moignet, A.; Lamy, T. Latest Advances in the Diagnosis and Treatment of Large Granular Lymphocytic Leukemia. Am. Soc. Clin. Oncol. Educ. Book 2018, 38, 616-625. [CrossRef] [PubMed]

5. Calabretto, G.; Teramo, A.; Baril, G.; Vicenzetto, C.; Gasparini, V.R.; Semenzato, G.; Zambello, R. Neutropenia and Large Granular Lymphocyte Leukemia: From Pathogenesis to Therapeutic Options. Cells 2021, 10, 2800. [CrossRef]

6. Zambello, R.; Teramo, A.; Gattazzo, C.; Semenzato, G. Are T-LGL Leukemia and NK-Chronic Lymphoproliferative Disorder really two distinct diseases? Transl. Med. UniSa 2014, 8, 4-11. [PubMed]

7. Oshimi, K. Clinical Features, Pathogenesis, and Treatment of Large Granular Lymphocyte Leukemias. Intern. Med. 2017, 56, 1759-1769. [CrossRef]

8. Barilà, G.; Calabretto, G.; Teramo, A.; Vicenzetto, C.; Gasparini, V.R.; Semenzato, G.; Zambello, R. T cell large granular lymphocyte leukemia and chronic NK lymphocytosis. Best Pract. Res. Clin. Haematol. 2019, 32, 207-216. [CrossRef] [PubMed]

9. Muñoz-García, N.; Jara-Acevedo, M.; Caldas, C.; Bárcena, P.; López, A.; Puig, N.; Alcoceba, M.; Fernández, P.; Villamor, N.; Flores-Montero, J.A.; et al. STAT3 and STAT5B mutations in T/NK-cell chronic lymphoproliferative disorders of large granular lymphocytes (LGL): Association with disease features. Cancers 2020, 12, 3508. [CrossRef]

10. Koskela, H.L.M.; Eldfors, S.; Ellonen, P.; Van Adrichem, A.J.; Kuusanmäki, H.; Andersson, E.I.; Lagström, S.; Clemente, M.J.; Olson, T.; Jalkanen, S.E.; et al. Somatic STAT3 mutations in Large Granular Lymphocytic Leukemia. N. Engl. J. Med. 2012, 366, 1905-1913. [CrossRef] [PubMed]

11. Clemente, M.J.; Wlodarski, M.W.; Makishima, H.; Viny, A.D.; Bretschneider, I.; Shaik, M.; Bejanyan, N.; Lichtin, A.E.; His, E.D.; Paquette, R.L.; et al. Clonal drift demonstrates unexpected dynamics of the T-cell repertoire in T-large granular lymphocyte leukemia. Blood 2011, 118, 4384-4393. [CrossRef]

12. Novikov, N.D.; Griffin, G.K.; Dudley, G.; Drew, M.; Rojas-Rudilla, V.; Lindeman, N.I.; Dorfman, D.M. Utility of a simple and robust flow cytometry assay for rapid clonality testing in mature peripheral T-Cell lymphomas. Am. J. Clin. Pathol. 2019, 151, 494-503. [CrossRef] [PubMed]

13. Muñoz-García, N.; Lima, M.; Villamor, N.; Morán-plata, F.J.; Barrena, S.; Mateos, S.; Caldas, C.; Balanzategui, A.; Alcoceba, M.; Domínguez, A.; et al. Anti-TRBC1 Antibody-Based Flow Cytometric Detection of T-Cell Clonality: Standardization of Sample Preparation and Diagnostic Implementation. Cancers 2021, 13, 4379. [CrossRef] [PubMed]

14. Shi, M.; Olteanu, H.; Jevremovic, D.; He, R.; Viswanatha, D.; Corley, H.; Horna, P. T-cell clones of uncertain significance are highly prevalent and show close resemblance to T-cell large granular lymphocytic leukemia. Implications for laboratory diagnostics. Mod. Pathol. 2020, 33, 2046-2054. [CrossRef] [PubMed]

15. Horna, P.; Shi, M.; Olteanu, H.; Johansson, U. Emerging role of $t$-cell receptor constant $\beta$ chain- 1 (TRBC1) expression in the flow cytometric diagnosis of t-cell malignancies. Int. J. Mol. Sci. 2021, 22, 1817. [CrossRef] [PubMed]

16. Shi, M.; Jevremovic, D.; Otteson, G.E.; Timm, M.M.; Olteanu, H.; Horna, P. Single Antibody Detection of T-Cell Receptor $\alpha \beta$ Clonality by Flow Cytometry Rapidly Identifies Mature T-Cell Neoplasms and Monotypic Small CD8-Positive Subsets of Uncertain Significance. Cytom. Part B Clin. Cytom. 2020, 98, 99-107. [CrossRef] [PubMed]

17. van Dongen, J.J.; Lhermitte, L.; Böttcher, S.; Almeida, J.; van der Velden, V.H.; Flores-Montero, J.; Rawstron, A.; Asnafi, V.; Lécrevisse, Q.; Lucio, P.; et al. EuroFlow antibody panels for standardized n-dimensional flow cytometric immunophenotyping of normal, reactive and malignant leukocytes. Leukemia 2012, 26, 1908-1975. [CrossRef] [PubMed]

18. Kalina, T.; Flores-Montero, J.; van der Velden, V.H.J.; Martin-Ayuso, M.; Böttcher, S.; Ritgen, M.; Almeida, J.; Lhermitte, L.; Asnafi, V.; Mendonça, A.; et al. EuroFlow standardization of flow cytometer instrument settings and immunophenotyping protocols. Leukemia 2012, 26, 1986-2010. [CrossRef] [PubMed]

19. EuroFlow. Available online: https:/ / www.euroflow.org/ (accessed on 16 December 2021).

20. Langerak, A.W.; Groenen, P.J.T.A.; Brüggemann, M.; Beldjord, K.; Bellan, C.; Bonello, L.; Boone, E.; Carter, G.I.; Catherwood, M.; Davi, F.; et al. EuroClonality/BIOMED-2 guidelines for interpretation and reporting of Ig/TCR clonality testing in suspected lymphoproliferations. Leukemia 2012, 26, 2159-2171. [CrossRef]

21. van Dongen, J.J.M.; Langerak, A.W.; Brüggemann, M.; Evans, P.A.S.; Hummel, M.; Lavender, F.L.; Delabesse, E.; Davi, F.; Schuuring, E.; García-Sanz, R.; et al. Design and standardization of PCR primers and protocols for detection of clonal immunoglobulin and T-cell receptor gene recombinations in suspect lymphoproliferations: Report of the BIOMED-2 concerted action BMH4-CT98-3936. Leukemia 2003, 17, 2257-2317. [CrossRef]

22. Langerak, A.W.; Beemd, V.D.; Wolvers-tettero, I.L.M.; Boor, P.P.C.; Van Lochem, E.G.; Hooijkaas, H.; Van Dongen, J.J.M. Molecular and flow cytometric analysis of the VB repertoire for clonality assessment in mature TCRab T-cell proliferations. Blood 2001, 98, 165-174. [CrossRef] 
23. Lima, M.; Almeida, J.; Teixeira, M.D.A.; Alguero, M.D.C.; Santos, A.H.; Balanzategui, A.; Queirós, M.L.; Bárcena, P.; Izarra, A.; Fonseca, S.; et al. TCR $\alpha \beta+/ C D 4+$ large granular lymphocytosis: A new clonal T-cell lymphoproliferative disorder. Am. J. Pathol. 2003, 163, 763-771. [CrossRef]

24. Morice, W.G.; Kimlinger, T.; Katzmann, J.A.; Lust, J.A.; Heimgartner, P.J.; Halling, K.C.; Hanson, C.A. Flow Cytometric Assessment of TCR-V $\beta$ Expression in the Evaluation of Peripheral Blood Involvement by T-Cell Lymphoproliferative Disorders: A Comparison with Conventional T-Cell Immunophenotyping and Molecular Genetic Techniques. Am. J. Clin. Pathol. 2004, 121, 373-383. [CrossRef] [PubMed]

25. Lima, M.; Teixeira, M.D.A.; Queirós, M.L.; Santos, A.H.; Gonçalves, C.; Correia, J.; Farinha, F.; Mendonça, F.; Soares, J.M.N.; Almeida, J.; et al. Immunophenotype and TCR-V $\beta$ repertoire of peripheral blood T-cells in acute infectious mononucleosis. Blood Cells Mol. Dis. 2003, 30, 1-12. [CrossRef] 